\title{
Raumkonstellationen in Jan Faktors Roman "Schornstein"
}

\section{Constellations of Space in Jan Faktors novel 'Schornstein'}

\author{
Karl-Heinz Gmehling
}

\begin{abstract}
Jan Faktor is a Czech-German writer and translator who was born in Prague in 1951 and moved to East Berlin in 1978 following his marriage to Annette Simon, the daughter of Christa Wolf. The move resulted in a literary language change. Factor's works address issues such as migration, homeland, foreigners, outsiders, identity and the Holocaust. Using the theoretical approaches of the "spatial turn" (Bachmann-Medick 2006), the present article examines the question of which literary spaces are constructed in which way in his first novel "Schornstein" (2005), how they change and which functions they fulfill. The analysis of literary spaces includes both the 'concrete narrated spaces' (Frank 2017), but does not exclude the metaphorical use of the term 'space'.
\end{abstract}

\section{Keywords}

Jan Faktor, Spatial Turn, Space, Outsider, Hybridity, Transit, Borders, Holocaust. 


\section{Einleitung}

Jan Faktor ist im Prag der Fünfziger- und Sechziger Jahre des letzten Jahrhunderts aufgewachsen. Nachdem er ein Studium der Datenverarbeitung nach drei Semestern abgebrochen hatte, nahm er verschiedene Arbeitsverhältnisse auf. Unter anderem arbeitete er als Programmierer in einem Prager Rechenzentrum und zwei Jahre auch als ,Lastenträger' in den Bergen der Slowakei. Das gewaltsame Ende des ,Prager Frühling“ war für Faktor ein einschneidendes Erlebnis. Das politische, kulturelle und gesellschaftliche Klima in der Tschechoslowakei wurde für ihn so belastend, dass er nach seiner Heirat mit Annette Simon, der Tochter von Christa Wolf, in die DDR emigrierte. In Ost-Berlin arbeitete er als Übersetzer, Kindergärtner und Schlosser. ${ }^{1}$ Bis 1989 war Faktor dort fast ausschließlich in der alternativen Literaturszene am Prenzlauer Berg tätig. Mit seinem ersten Roman „Schornstein“, der im Jahre 2005 im Verlag Kiepenheuer \& Witsch erschien und für dessen Manuskript er den „Alfred-Döblin-Preis“ bekam, schaffte Faktor den literarischen Durchbruch.

In „Schornstein“ geht es um den gleichnamigen Protagonisten², der mit seiner Frau Anne im Berlin der Nachwendezeit lebt. Schornstein hat einen Herzinfarkt überstanden und sich mit der dabei zufällig entdeckten, sehr seltenen Stoffwechselkrankheit abgefunden. Als man ihm eines Tages aus für ihn unverständlichen Gründen die Bezahlung der teuren Therapie verweigert, beginnt der Protagonist seinen Kampf gegen das (Gesundheits-) System. Er konsultiert Ärzte und Wissenschaftler, sammelt Informationen über seine noch wenig erforschte Krankheit und ermittelt andere Betroffene. Schließlich muss Schornstein krankheitsbedingt seine Arbeitsstelle kündigen, er findet aber noch die Zeit, sich um Außenseiter der Gesellschaft - einige Trinker aus seinem Wohnviertel oder die ziemlich verwahrloste Wohnungsnachbarin Frau Schwan - zu kümmern. Sein monatelanger Kampf führt ihn schließlich in eine existenzielle Krise, die ihn physisch und psychisch schwer belastet und seine Frau an den Rand der Verzweiflung bringt. Die professionelle Hilfe eines Psychiaters und die Liebe seiner aufopferungsvollen Frau bringen schließlich die, Wende'.

Im Folgenden wird eine raumnarratologische Analyse des Romans durchgeführt, durch die eruiert werden kann, welche konkreten und metaphorischen Räume im Roman wie dargestellt werden, welchen Charakter sie haben und welche Funktionen diese Räume erfüllen. Dabei werden näher die Raumkonstellationen als Makroräume mit weltrealem Pendant und Mikroräume ohne weltreales Pendant, als Räume mit marginalem, hybridem und heterotopem Charakter sowie als Räume der Grenzen und Grenzüberschreitungen, der Macht und Ohnmacht und nicht zuletzt als Räume der Sprache untersucht. Des Weiteren soll neben den Raumkonstellationen auch auf die narrative Darstellung und die Semantik des erzählen Raumes fokussiert werden. Dazu werden verschiedene raumtheoretische Ansätze verwendet.

1 Hier wird der autobiografische Charakter des Romans deutlich, denn einige dieser Tätigkeiten übt auch der Protagonist im Roman „Schornstein“ aus.

2 Der Vorname des Protagonisten bleibt unbekannt. 


\section{Raumtheoretische Ansätze und ihre Grenzen}

Der sogenannte „spatial turn“ Ende der 1980er-Jahre markierte eine wissenschaftliche Wende bezüglich der Raumvorstellungen. Der US-amerikanische Stadtplaner und Namensgeber dieses Begriffes Edward Soja diagnostizierte in den 1980ern eine zunehmende Verräumlichung des Denkens (vgl. Soja in Hallet 2009: 58) und die Literaturwissenschaftlerin Bachmann-Medick spricht in ihrem Werk ,Cultural Turns“ vom „Raum als Analysekategorie“ (Bachmann-Medick 2009: 303) und von einem dazu notwendigen „raumbezogenen Denken“ (Ebd.: 292). Raum wird nicht mehr wie bisher als (neutraler) Behälter, innerhalb dessen sich (historische) Ereignisse abspielen, angesehen, sondern als „gesellschaftlich produzierter Raum“ (Soja, 1991: 75). Die Soziologin Martina Löw, deren Raumtheorie „sich mittlerweile als ,Basismonographie des spatial turn in der deutschsprachigen Soziologie“ etabliert hat“ (Wilhelmer 2015: 20) bezeichnet die beiden konkurrierenden Raumvorstellungen als „absolutistisch“ (Raum als Behälter) und „relativistisch“ (Raum als gesellschaftlich produzierter Raum, generiert aus der Perspektive einer relativen Beobachterposition) (Löw in Wilhelmer 2015: 20). Löw spricht in diesem Zusammenhang von einem „relationalen“ Raumverständnis und versucht dadurch die beiden konkurrierenden Raumvorstellungen zu einem einzigen, fruchtbaren Raummodell weiter zu entwickeln. „Raum“ ist nach Löw eine „relationale (An)Ordnung von Lebewesen und sozialen Gütern an Orten“ (Löw in Wilhelmer 2015: 26).

Nützlich für die Analyse des Textes sind auch andere, ältere sowie jüngere raumnarratologische Theorien: die Raumsemantik nach Jurij Lotman (Lotman 1972, 2010), in der der Begriff der Grenze bzw. der Grenzüberschreitung eine wichtige Rolle spielt, die Theorie der „Transit-Orte“ nach Wilhelmer (vgl. Wilhelmer 2015), das Phänomen der ,Hybridität‘ (angelehnt an Homi Bhabhas Konzept 1994), das Konzept der „Heterotopien“ (nach Michel Foucault 2006) oder die ,Räume der Macht‘ (nach Bourdieu 1999).

Im vorliegenden Roman erfolgt die Analyse der literarischen Raumdarstellung in Grundzügen nach dem Analysemodell von Caroline Frank (2017), welches das bis dato aktuellste raumnarratologische Analysemodell in der Literaturwissenschaft ist. Frank untersucht die „konkreten Räume der erzählten Welt“ (Frank, 2017: 71) und ihre Analyse vollzieht sich in drei Schritten: 1) Auswahl und Kombination von (Teil-) Räumen, 2) Narrative Darstellung der Räume 3) Semantik der erzählten Räume. Frank lässt die metaphorische Verwendung des Begriffes ,Raum‘ in ihrer Analyse (bedauerlicherweise) außen vor. Diese wird meines Erachtens für eine Raumanalyse von interkultureller Literatur jedoch als wesentlich erachtet und sie soll dementsprechend bei der Analyse der konkreten Räume der erzählten Welt immer ,mitgedacht‘ werden. Auf diese Weise wird versucht, beide Raumarten - konkrete und metaphorische Räume - miteinander zu verschränken und dadurch die metaphorischen Räume in Franks Modell zu integrieren. Um eine Überfrachtung mit metaphorischen Räumen, die prinzipiell schwer kategorisierbar sind, zu vermeiden, wird in der vorliegenden Untersuchung von vornherein eine Einschränkung vorgenommen: Es wird die Analyse auf diejenigen metaphorischen Räume festgelegt, die bereits bei der Analyse der konkreten Räume in Erscheinung 
traten $^{3}$ und auf diejenigen, die bei den oben genannten ,Raum-Theoretikern“ explizit angesprochen worden sind.

\section{Raumkonstellationen im Roman „Schornstein”}

$\mathrm{Zu}$ Beginn der raumnarratologischen Analyse bietet es sich an, die konkreten Räume der erzählten Welt im Roman „Schornstein“ (weiter als „S“ abgekürzt) vorzustellen, die einen realweltlichen Bezug haben. Hierbei kann erst einmal zwischen Makro- und Mikroräumen unterschieden werden.

\subsection{Makroräume - mit realweltlichem Pendant}

Die Handlung des Romans spielt im Berlin der Nach-Wendezeit, genauer gesagt im Ostberliner Ortsteil Pankow. Pankow, speziell der Prenzlauer Berg, ist durch seine ausgeprägte ,Untergrund-Szene‘ bekannt. Viele Künstler, insbesondere Schriftsteller aber auch ,Aussteiger" wohn(t)en hier, autobiographische Bezüge sind für den informierten Leser bereits zu Beginn des Romans unübersehbar. ${ }^{4}$ Weitere markante Makroräume mit realweltlichem Pendant bzw. narratologischer Bedeutung ${ }^{5}$ werden im Roman nicht aufgerufen. Die Wahl von Berlin als Makroraum, in dem die Handlungen verortet werden, hat (auch) Auswirkungen auf den Fortgang der Handlung, da Berlin bekanntlich jahrzehntelang räumlich-geografisch und dementsprechend auch gesellschaftlich und politisch geteilt war und die Stadt erst relativ kurz vor Beginn der erzählten Zeit wieder Hauptstadt des wiedervereinigten Deutschland geworden ist. Schornstein ist die einzige Figur im Roman, die aus dem ,Westen', sprich Westdeutschland, kommt, alle anderen Figuren kommen aus dem ,Osten': Ostberlin, Ostdeutschland oder -europa. In der wiedervereinigten Haupt-Stadt Berlin liegt sowohl die Zentrale der „Kassenärztlichen Vereinigung“, also des ,Hauptgegenspielers“ von Schornstein, als auch der Sitz des „Bundesausschuss Qualitätssicherung und -standard“, der für Schornstein die „eigentliche Schaltstelle“ bzw. die „Quelle der Macht“ (alles S. 251) darstellt. Berlin fungiert also gleich mehrfach als ein Zentrum.

3 Räume können den gleichen oder einen ähnlichen ,konkreten“ und ,metaphorischen“ Charakter haben, z. B. ein marginaler Raum am Rande einer Stadt.

4 Jan Faktor wohnt seit seiner Übersiedlung nach Deutschland ebenfalls in Pankow und war jahrelang in der Literaturszene des Prenzlauer Berges aktiv.

5 Anne fliegt einmal für eine Woche nach Nigeria, um eine psychotisch gewordene Freundin zu besuchen. Dies spielt für den Fortgang der Handlung keine herausragende Rolle. 


\subsection{Mikroräume - ohne realweltliches Pendant}

Bei den Mikroräumen der erzählten Welt handelt es sich durchwegs um imaginierte Räume, bei denen der Leser ${ }^{6}$ nicht festzustellen vermag, ob es realweltliche Bezüge gibt. Trotzdem haben diese Räume auch einen gewissen realweltlichen Bezug, da sie alltagsweltlichen Charakter haben. Es sind vorwiegend Innen-Räume von Wohnungen: Zimmer, Kellerräume, Treppenhäuser oder ein Dachgeschoss. Eine genauere Verortung dieser Räume, sei es durch Angabe von Straßennamen oder Adressen, gibt es nicht. Auch Behandlungs- und Wartezimmer in Krankenhäusern und Arztpraxen werden zu relevanten Räumen im Leben des ,dauerkrank' gewordenen Protagonisten. Eine besonders wichtige Rolle spielt der menschliche Körper, insbesondere der des Protagonisten. Auf diesen Körper-Raum als sozusagen kleinsten Mikroraum oder Nah-Raum des Protagonisten wird weiter unten gesondert eingegangen, da er von besonderer Relevanz ist. Schließlich spielt noch ein ,externer‘ Mikroraum, ein verkommenes Rondell an einem Supermarkt eine herausragende räumliche und soziale Rolle. Diese kurze Übersicht über die Raumauswahl der konkreten Makro- und Mikroräume deutet insgesamt betrachtet auf eine typische Ostberliner Wohnlandschaft und -situation kurz nach der ,Wende“ hin. Wie kann man nun diese Räume genauer unterscheiden und charakterisieren?

\subsection{Räume mit marginalem, hybridem ${ }^{7}$ und heterotopem Charakter}

Bereits von Beginn an wird deutlich, dass einige der oben genannten Mikroräume auch metaphorische, hier in erster Linie marginale Bedeutung haben. Das genannte Rondell ist ebenso ein marginaler Raum wie der Keller und das Dachgeschoss des Mietshauses. Die Räume liegen sowohl konkret als auch metaphorisch ,am Rand': im obersten Stock des Mietshauses, im Keller desselben oder am Rande „einer kleinen vernachlässigten Parkanlage“ (S. 84), d. h. am Rande der ,zivilisierten“ Natur und gleichzeitig auch am Stadt-Rand, als dem ,Rand der Zivilisation‘. Wie bereits im Klappentext angekündigt, handelt dieser Roman „vom brüchigen Rand der Gesellschaft“. Und an diesen Rändern halten sich nicht nur die Trinker als symbolische, gesellschaftliche Verlierer der NachWende-Zeit auf, sondern auch die pubertierenden Jugendlichen, die sich ihre eigenen Treffpunkte und Aufenthalts-Räume im Keller oder Dachgeschoß schaffen.

Ein besonders markantes Beispiel eines marginalen, gleichzeitig aber auch dynamischen und hybriden Raumes ist die Wohnung von Frau Schwan. Sie ist Witwe und gehbehindert, ihre Wohnung liegt im Parterre des Mietshauses. Frau Schwan hat „ein offenes Haus für Menschen, die es im Leben nicht leicht hatten“ (S. 120), deshalb fungiert ihre Wohnung

6 Im Folgenden wird aus Gründen der besseren Lesbarkeit ausschließlich die männliche Form verwendet. Sie bezieht sich auf alle lesenden Personen, unabhängig vom Geschlecht.

7 Hybrid wird hier in erster Linie in der Wortbedeutung von "gemischt“ verstanden. Es handelt sich nicht wie bei Homi Bhabha um ein Aufeinandertreffen von Kulturen, wodurch sich ein ,Zwischenraum“ oder ,dritter Raum`öffnet, sondern um eine produktive Kontaktzone, in der unterschiedliche soziale und gesellschaftliche Schichten aufeinandertreffen. 
auch als heimlicher Treffpunkt der Trinker vom Rondell bzw. als deren zeitweilige Übernachtungsmöglichkeit. Weil sich Letztere nur vorübergehend, für Stunden oder Tage, in ihrer Wohnung aufhalten, bekommt diese auch einen dynamischen und gleichsam transitären Charakter. Schornstein - anfangs als (noch) arbeitender Angestellter einer Werbeagentur sozusagen ein Vertreter der ,sozialen Mitte“- darf als „absoluter Lieblingsmieter“ (S. 77) von Frau Schwan auch ihre Wohnung betreten. Das eindringlichste ,Beschreibungsmerkmal der Wohnung, das sie auch olfaktorisch zu einem besonderen Raum macht, ist der Gestank, der von ihr ausgeht und der sich übers Treppenhaus im ganzen Haus verteilt. Das diesbezügliche Auftreten und Verhalten von Frau Schwan gegenüber ihren Mitbewohnern erinnert gar an einen heterotopen Raum in Sinne Foucaults. ${ }^{8}$

Das Rondell am Rande des Parkes als zweiter temporärer aber öffentlicher Aufenthaltsraum der Trinker hat nicht nur einen marginalen Charakter, sondern auch einen transitären und in zweierlei Hinsicht dynamischen Charakter. Es sollte ursprünglich als „Stück Gartenkunst“ (S. 85) den Zugang zum Park verschönern. War das Rondell vor der ,Wende“ ein schöner Treffpunkt alter Damen zum Plausch gewesen (vgl. S. 86), so ist es mittlerweile schäbig und heruntergekommen und wird von den meisten Anwohnern gemieden, weil man die „intim-schmuddelige Atmosphäre des leicht bemosten Plätzchens“ (S. 86) nicht stören wolle. Dies gilt aber nur für die warme Jahreszeit, im Winter übernehmen junge Frauen mit ihren Kindern oder Omas und Ortsfremde zeitweise diesen Raum (vgl. S. 10). Das Rondell ändert(e) somit seine Gestalt, Funktion und seine Atmosphäre nicht nur stadthistorisch gesehen, sondern auch periodisch mit dem Lauf der Jahreszeiten.

Ein Mikroraum, dem eine besonders relevante, wenn nicht die wichtigste Bedeutung im Leben des Protagonisten zukommt, ist die Arztpraxis des Psychiaters Dr. Brakwart. Wie die Wohnung von Frau Schwan, so wird auch dieser Raum ausführlich beschrieben. Er kann ebenfalls als transitärer, dynamischer und gleichzeitig mehrfach hybrider Raum identifiziert werden. Das kündigt sich bereits beim ersten Anruf Schornsteins in der Praxis an. Die Sprechstundenhilfe, die, wie sich später herausstellt, in diesem Warteraum arbeitet, wirkt am Telefon zunächst wie „ein Engel“ (S. 178). Beim zweiten Anruf - nur wenige Minuten später, aber in der Mittagspause - ist sie wie ausgewechselt: kurz angebunden und unfreundlich wirkt sie auf Schornstein wie ein „Todesengel“ (S. 179) und er hasst „diese Vorzimmerkröte von nun wie die Pest“ (S. 179). Als Schornstein schließlich das Haus des Arztes aufsucht, gerät er beim Eintreten versehentlich in die Privaträume statt in die Praxis des Arztes. Wiederholt kommt es im Haus des Arztes zu einer ungewollt-peinlichen Verwechslung von privaten und öffentlichen Räumen seitens

8 Frau Schwan achtet akribisch darauf, dass ihre Wohnung nur Leute betreten, die sie mag und die sie deshalb zu sich einlädt: einige der älteren Trinker und Schornstein. Für alle anderen Figuren, auch den Hausmeister oder die Putzfrau des Hauses, ist die Wohnung tabu. Frau Schwan versperrt ihren Wohnungseingang „breit und unüberwindlich wie eine Panzersperre“ (S.117). Dies erinnert an die für Heterotopien typischen Öffnungs- und Schließungsrituale. Zudem werden in ihrer Wohnung mehrere Räume bzw. Raumarten zueinander in Beziehung gesetzt, die eigentlich nicht vereinbar sind: Wohnraum, Raum für Zusammenkünfte, Tabu-Raum und Asylraum. Als Letzterer wird in der Wohnung auch mit der herkömmlichen Zeitstruktur gebrochen, was ebenfalls ein Kennzeichen von Heterotopien ist. (vgl. Foucault in Günzel 2006: 321ff). Allerdings ist Frau Schwans Wohnung kein institutioneller Ort, sondern privat und in diesem Sinne keine Heterotopie im Sinne Foucaults. 
des Protagonisten. Dies passiert auch deshalb, weil die Räume teilweise nur durch einen Vorhang getrennt sind (vgl. S. 181). Dadurch wird insgesamt die dynamische Hybridität dieser Räume deutlich, welche letztendlich ,Grenzüberschreitungen“ seitens der Patienten förmlich herausfordert. Besonders deutlich empfindet Schornstein diese Hybridität im riesigen Wartezimmer.

Was sich dort außerdem befand, gab mir noch mehr zu denken: ein riesiger Schreibtisch, auf dem sich Patientenakten stapelten. [...] ich überlegte kurz, was der Schreibtisch, dieser sozusagen „patientennahe Arbeitsplatz" hier zu suchen hatte, und war mir bald sicher, daß ich richtig lag: Die offenherzige laute Plaudertasche von Schwester thronte hier und führte alle höchst vertraulichen Gespräche mit den Patienten - und nicht nur die telefonischen - vor der versammelten Wartemannschaft. (S.180)

Das Wartezimmer - eigentlich ein klassischer Transitraum - tritt hier als besonders skurrile Mischung aus öffentlichem und privatem Raum in Erscheinung. Datenschutz bzw. die ärztliche Schweigepflicht werden hier scheinbar demonstrativ unterlaufen. Als Schornstein die Sprechstundenhilfe später live an ihrem Schreibtisch erlebt, sieht er sich in seinen Befürchtungen bestätigt. Sie zieht „hier vor all den verzweifelten und kaputten Leuten eine Lifeshow ab. [...]. Im Grunde lief hier nebenbei ein ziemlich abartiger Amüsierbetrieb“ (S. 180). Das Wartezimmer wird nicht nur zu einer peinlichen Mischung aus privatem und öffentlichem Raum, es dient gleichzeitig als Bühne für die Auftritte der Schwester und als unfreiwilliger, aufgezwungener Zuschauer- bzw. Zuhörerraum der dort wartenden Patienten. Dies verleiht auch diesem Raum heterotope Züge. ${ }^{9}$

\subsection{Grenzen und Grenzüberschreitungen}

Der Protagonist ist Jude, fühlt sich aber von klein auf eher mit anderen „Minderheiten oder Gestalten“ (S. 30) verwandt. In seiner Wohngegend seien dies „neben einigen Vietnamesen die Alkoholkranken und Obdachlosen" (S. 30). Zu diesen gehören für Schornstein „in erster Linie [...] Frau Schwan und die mit ihr assoziierten Alkoholjuden“ (S. 30). Schornstein selbst ist zunächst kein Außenseiter, wird aber infolge seiner extrem seltenen Krankheit und der damit verbundenen persönlichen und beruflichen Folgen allmählich dazu. Gleichzeitig sucht er die Nähe der von ihm geschätzten Minderheiten. So betritt er als einziger die oben erwähnten marginalen Räume: das Rondell und Frau Schwans Wohnung. Schornstein fungiert somit als eine Art Vermittler: zwischen den Figuren, die den sozialen Rand der Gesellschaft verkörpern, und der ,gesellschaftlichen Mitte', in der sich auch seine Frau und der Hausmeister verortet sehen (vgl. S. 75). Bei dieser ,Vermittlertätigkeit' überschreitet Schornstein mehrere sichtbare und unsichtbare, konkrete und metaphorische Grenzen.

Zum einen überschreitet er (unsichtbare) sozialgesellschaftliche Grenzen. Als er eines Tages Frau Schwan im Rondell bei den Trinkern entdeckt, „mitten in der trüben

9 Das Theater ist nach Foucault eine klassische Heterotopie. (vgl. Foucault in Günzel 2006: 324) 
Männerrunde“ (86), ist das zwar ein „kleiner Schock“ (S. 87) für ihn, aber er geht trotzdem ins Rondell zu den Trinkern und beginnt, vermittelt durch Frau Schwan, ein Gespräch mit ihnen. Er ist hier nicht nur der einzige, der aus dem ,Westen' kommt, auch die Wortwahl bei der Beschreibung seiner beruflichen Tätigkeit - „Printmedien“ (S. 88) - unterscheidet ihn von den anderen. Er merkt, dass er sprachliche bzw. intellektuelle Grenzen überschritten hat, die Trinker um Frau Schwan sehen ihm dies aber nach und akzeptieren ihn. Als Folge dieser, für Schornstein ,erfolgreichen' Grenzüberschreitung hat er zu Frau Schwan und den Trinkern ein anderes, engeres Verhältnis, was sich auch in wiederholten Hausbesuchen der Trinker bei Schornstein zeigt

Deutlich markiert werden auch konkrete Grenzen zwischen privaten und öffentlichen Räumen. Dies sieht man besonders an den Schilderungen der Wohnungen und dem Verhalten ihrer Bewohner. Der Hausmeister Herr Krabow richtet an der ,Grenze‘ seiner Wohnung - innen an seiner Wohnungstür - eine Art ,Horchposten' (vgl. S. 35) ein, von dem aus er das ganze Treppenhaus außen überwachen will, um jugendliche ,Störenfriede aus dem Haus fernzuhalten. Frau Schwan steht zwar oft auf der Schwelle ihrer offenen Wohnungstür, um sich über Neuigkeiten im Haus auszutauschen, verweigert aber strikt allen Mitbewohnern, außer wie gesagt Schornstein oder den Trinkern den Zugang zu ihrer Wohnung. Auch an Schornsteins räumlichen Verhalten erkennt man deutlich, dass er Grenzen zwischen seinen privaten und den öffentlich zugänglichen Räumen in seiner Wohnung zieht. Er lässt zwar manchmal neugierige Trinker in seine Wohnung eintreten, achtet aber darauf, dass sie nicht bis ins Schlafzimmer vordringen, „das tabu bleiben würde“ (S. 239). Als es einem der Trinker einmal doch gelingt, diese „Schwelle“ (S. 240) zu überschreiten, ist Schornstein „sprachlos“(S. 240). Dass nicht nur im Wartezimmer des Psychiaters ,Schamgrenzen` überschritten, ja die Überschreitung geradezu inszeniert wird, sondern auch innerhalb der Wohnung von Schornstein unsichtbare aber zeitweilig unüberwindbare (Scham-) Grenzen wirken, wird in der Szene deutlich, als er sich in seinem Arbeitszimmer, dem hintersten Zimmer der Wohnung regelrecht eingesperrt fühlt, weil seine Frau im davor liegenden Wohnzimmer, einen geschäftlichen Besuch empfängt. (vgl. 81). ${ }^{10}$

Auch die Grenze zwischen Gesundsein und Kranksein wird thematisiert. Für den Protagonisten und seine Frau als auch den Leser wird sie im Laufe der Geschichte immer verschwommener. Anne bezweifelt zunehmend, dass es ihrem Mann wirklich so schlecht gehe, er wiederum ,spielt‘ allmählich mit seinem Krank-Sein und denkt zuweilen, dass es ihm schon „wieder schlechter gehen dürfte“ (S. 227). Schließlich sinniert Schornstein, ob er nicht bereits im Begriff ist, die Grenze zum Wahnsinn zu überschreiten (vgl. 174).

Bei seiner Suche nach Unterstützern muss Schornstein erfahren, dass auch bei (hilfsbereiten) Politkern „ihre Macht Grenzen hat“ (S. 64). Dies verweist auf einen anderen konkreten und gleichzeitig metaphorischen Raum, den Raum der Macht, wie ihn bereits Bourdieu mit seiner Feldtheorie (vgl. Dünne 2015, 243) als auch Foucault (vgl. Foucault 2017) aus unterschiedlicher Perspektive beschrieben und analysiert haben. Macht durch-

10 Dass bei der anschließenden Beschreibung von Schornsteins „Fäkalaktion“ für den einen oder anderen Leser auch Grenzen des literarischen Geschmacks ausgelotet bzw. überschritten werden, spiegelt sich in nahezu allen Rezensionen wieder. 
dringt und dominiert im Roman viele Räume, deshalb soll auf diesen metaphorischen Raum im Folgenden genauer eingegangen werden.

\subsection{Räume der Macht - Ohnmacht}

Bei seinem monatelangen Kampf um die (Weiter-) Zahlung der teuren Therapie kontaktiert Schornstein alle möglichen Institutionen des „gesundheitsbürokratische[n] Genehmigungs- und Verweigerungssystem[s]“ (S. 40), um die ihm „völlig schleierhaften Kompetenzstrukturen“ (S. 43) zu durchschauen. Schließlich ist für ihn die „KASSENÄRZTLICHE VEREINIGUNG“ (S. 16), welche ihren Sitz in der Haupt-Stadt Berlin hat, der eigentliche Gegner. Sie sei kein „Patientenschutzbund“, sondern eher eine „Kampfmaschine der niedergelassenen Ärzte“ und gleichzeitig „eine Welt für sich“ (alles S. 52), gegen die man nicht ankomme. Für Normalsterbliche sei sie ein Dschungel ohne jegliche Kontrolle. Schornstein recherchiert sogar die Geschichte dieser Institution und erfährt dadurch, dass sie in der Zeit des Nationalsozialismus in Deutschland mit einer zwielichtigen Organisationsstruktur und Machtfülle ausgestattet worden sei, die bis heute mehr oder weniger unverändert beibehalten worden ist. Dass eine Ärztelobby eine so große Macht in einem demokratischen Staat habe, sei ungewöhnlich. (vgl. 256f.). Laut Renata Cornejo ist es die nahezu kafkaeske „Ohnmacht eines Individuums angesichts eines Machtapparats, der nicht durchschaubar, nicht erklärbar, nicht greif- und begreifbar ist" (Cornejo 2010: 381), die ein zentrales Thema dieses Romans ausmacht. Auf einen konkreten erzählten Raum heruntergebrochen zeigt sich die Macht - und ihr Missbrauch - paradigmatisch im Wartezimmer des Arztes. Die Sprechstundenhilfe dominiert den Raum von ihrer zentralen Position am Schreibtisch aus und drangsaliert sowohl die Patienten am Telefon als auch die im Raum Anwesenden. Doch die Ohnmacht des Protagonisten ist immer nur eine vorübergehende. Zu seinem Glück findet er zahlreiche Helfer, unter anderem einen privaten Geldgeber namens Guido, dessen ,finazielle Macht‘ es ihm ermöglicht, seine Therapie wieder aufzunehmen, weiter zu leben und zu kämpfen. Schornsteins „Hauptwaffe‘ in seinem Kampf um Gerechtigkeit ist jedoch die Sprache.

\subsection{Raum der Sprache - Kommunikation und ihre Grenzen}

Der Gebrauch der Sprache, sowohl die schriftliche als auch die mündliche Kommunikation, spielen im Leben und Alltag von Schornstein eine dominante Rolle. Das zeigt sich daran, dass Kommunikation in nahezu allen Situationen und bei allen Begegnungen mit anderen Figuren stattfindet bzw. thematisiert wird. Es gibt zahlreiche Dialoge, die - abgesehen von kurzen Unterbrechungen - auf mehreren Seiten in wörtlicher Rede wiedergegeben werden. ${ }^{11}$ Schornstein und seine Frau Anne haben auch beruflich mit

11 Bsp.: S. 54-56, S. 101-104, S. 169-173 oder S. 194-199! Auf über hundert Seiten des zweihundertachtzigseitigen Romans sind Dialoge in wörtlicher Rede zu finden. Hinzu kommen noch die in indirekter Rede wiedergegebenen Gespräche. 
Sprache zu tun. Er arbeitet bis zu seiner Erkrankung als Seniortexter bei einer Werbeagentur und Anne gestaltet als Graphikdesignerin Flyer, Plakate und Programmhefte (vgl. S. 59). Da sie beide von zuhause aus arbeiten, haben sie für ihre Wohnung ein spezielles, rein körpersprachliches Kommunikationsverhalten entwickelt (vgl. S. 61), um sich nicht gegenseitig von der Arbeit abzuhalten, Zudem telefoniert Schornstein nicht nur mit allen möglichen Ärzten und Krankenkassen, um sich zu informieren, sondern unterhält sich auch regelmäßig mit seinen Hausmitbewohnern. Sein insgesamt sehr reges Kommunikationsverhalten erstreckt sich sozusagen auf viele Räume. Als er Frau Schwan unvermittelt im Rondell antrifft, nutzt er die für ihn „einmalige Gelegenheit“ (S. 87), um mit den Trinkern ins Gespräch zu kommen. „Das Gespräch lief ganz gut an, ich konzentrierte mich nur auf Frau Schwan und schaute nicht nach links oder rechts. Man hörte uns zu. Falls es nötig sein sollte, war ich auf einen gesonderten Rückzug („Bin in Eile-“) vorbereitet"(S. 87).

Besonders ausgiebig und regelmäßig bespricht er sich (natürlich) mit seiner Frau Anne über seine Empfindungen und Zustände bezüglich seiner Krankheit. Aber mit fortlaufender Zeit und ausbleibender Besserung seines Gesundheitszustandes wird Anne sein nahezu exzessives Erzählbedürfnis zu viel. ${ }^{12}$ Schließlich einigt er (sic!) sich bezüglich seines Gesundheitszustandes auf den banalen Satz: „Mir geht es nicht gut“ (S. 174). Hier werden die Grenzen der Kommunikation deutlich. Sprache versagt als Mittel zur adäquaten Beschreibung persönlicher Empfindungen. ${ }^{13}$ Dass die Gespräche von Schornstein sehr bewusst wahrgenommen werden, zeigt sich besonders deutlich in seinen Gesprächen mit dem (ihm unsympathischen) Hausmeister Herrn Kabrow:

Das Quälende an Herrn Kabrow war [...], daß er neben seiner offensichtlichen Dummheit den Ehrgeiz besaß, alles auf eine dem Gesprächspartner angemessene höhere Ebene zu versetzen, die Dinge zu umschreiben, zu verallgemeinern - und sie dem Zuhörer eben ganz vornehm bildlich und didaktisch-genau nahezubringen. (S. 36)

Schornstein analysiert die Gespräche nicht nur nach deren Charakter, sondern auch nach deren Funktion. So will der Hausmeister bei seinen Gesprächen mit Schornstein „die Auflösung seiner Ausführungen nach Möglichkeit nie direkt aussprechen. Sein Gesprächspartner sollte es lieber selbst tun“ (S. 36). Auf diese Weise will er den Gesprächspartner als „mitverantwortlichen Urheber der eventuellen Problemlösung vorschieben“ (S. 36). Besonders auch gegenüber Ärzten und Rechtsanwälten, die Schornstein konsultiert, um Hilfe zu bekommen, zeigt sich die zentrale Bedeutung von Kommunikation. Die Zusammenarbeit mit dem Psychiater Dr. Brakwart, dem Mann, der ihm letzten Endes am meisten helfen kann, erstreckt sich über einen monatelangen Zeitraum gemeinsamer Sitzungen und zeigt dabei eine erstaunliche Entwicklung: die Beziehung zwischen

12 Das Erzählen ist für Schornstein so wichtig, dass es ihm nahezu egal ist, wer ihm zuhört. „Schon aus Zeitvertreib hätte ich meine neuen psychodeskriptiven Theorien gern jemandem offenbart - es hätte nicht unbedingt Anne sein müssen." (173f.).

13 Mehrfach muss Schornstein die Erfahrung machen, dass seine Gesprächspartner - insbesondere Anne, aber auch Ärzte - die Kommunikation abrupt und ohne Begründung beenden. 
Arzt und Patient, das Kommunikationsverhalten und das entsprechende Machtverhältnis kehren sich nahezu um. Schornstein fühlt sich dem Psychiater „mehr und mehr überlegen“" (S. 204), dieser tue ihm leid (vgl. S. 205) und letztendlich verlässt Schornstein „den armen Dr. Brakwart“(S. 210).

\section{Narrative Darstellung des erzählten Raumes}

Der Ich-Erzähler Schornstein erzählt aus seiner Erinnerung. ${ }^{14}$ In dreißig Episoden schildert er rückblickend, was vor zehn Jahren, als er dreiunddreißig Jahre alt war, in seinem Leben passierte (ein Herzinfarkt) und wie es ihm seitdem erging. Er erzählt im epischen Präteritum, durchgehend aus der Ich-Perspektive, also rein subjektiv. Es gibt wenige heteroreferentielle Bezüge, die Handlung spielt im Berlin der Nachwendezeit. Der erzählte Raum, insbesondere die konkreten Mikroräume, werden genauer beschrieben, über den ,Erzählraum` erfährt man nichts. Im Laufe der Erzählung kommt es wiederholt zu metatextlichen Einschüben.

Was ich im einzelnen unternahm und erfuhr, muß ich nicht alles unbedingt aufzählen; auch deswegen nicht, weil ich zu diesem Zeitpunkt immer noch nicht genug wußte und das gesundheitsbürokratische Genehmigungs- und Verweigerungssystem nicht verstand (S. 40).

Der Erzähler verweist auf einen abgekürzten Erzählakt, was eine emotionale Distanzierung zum erzählerischen Geschehen - seitens des Erzählers und auch des Rezipienten - beweist bzw. bewirken kann. Umgekehrt kann gerade dieser Hinweis auf den Erzählakt beim Leser eine ,Authentizität des Erzählten ${ }^{15}$ und eine emotionale Nähe zum Erzähler bewirken. Die Konstruiertheit des Erzählten und auch der Räume wird dadurch auf jeden Fall deutlich. Das Erzählen ist für den Protagonisten offensichtlich vom Erinnern und von seinem individuellen,Geschmack ‘ abhängig. So liegt für ihn beim Erzählen der Geschichten seiner Mutter „wieder alles klar und griffbereit da“ und er sieht plastisch vor sich, „wo und wie ihm verschiedene Geschichten erzählt worden waren“ (alles S. 208). An anderer Stelle bricht der Protagonist die Erzählung ab, da er „hygienisch problematische Dinge“ über sich „nicht unbedingt gern“ (alles S. 267) erzähle und dies nicht so wichtig in der Geschichte sei.

Die fast durchgehend verwendete Ironie in der Erzählerstimme evoziert ebenfalls eine Distanz zum Erzählten. So ist es nicht ersichtlich, wie Schornstein die obdachlosen Trinker einschätzt, wenn er sie als „Repräsentanten der alten Garde“ (S. 10), „örtliche Trinkerelite“ (S. 84) oder als „wirklich ruhige und ernsthafte Leute“ (S. 87) betitelt. Frau Schwan ist für ihn einerseits eine liebenswürdige alte Frau (vgl. S. 74ff), andererseits wirkt ihr Verhalten mehr als schrullig: Sie lüfte ihre Wohnung deshalb nicht, weil ansonsten die Abgase von

14 Auf den metaphorischen ,Erinnerungsraum‘, wie in Aleida Assmann vorschlägt (vgl. Assmann 2006), wurde in der Analyse verzichtet, da er im Roman eine zu allgemeine, umfassende Kategorie bildet.

15 Die in der Literatur angegebenen Rezensionen verweisen alle auf eine höchstwahrscheinlich stark autobiografische Färbung des Romans. 
der Straße ihre Wohnung - Schornstein nennt diese einmal auch, Gaskammer - verpesten würden (vgl. S. 76f). ${ }^{16}$ Schließlich ,bekennt" der Erzähler selbst, dass er in seiner Firma als Seniortexter „für die ironische Endkontrolle zuständig“ (S. 82) gewesen sei.

Zahlreiche kreative Wortschöpfungen und (scheinbar) paradoxe Gegensätze werden ebenfalls vor allem zur Ironisierung und Hyperbolisierung eingesetzt. So habe Schornstein an manchen Tagen eine „bettlägerische Form“ (S. 8), dann sei sein Körper „eine Art Muskel-, Knochen- und Sehnenerinnerung“ (S. 9). Der allseits unbeliebte Hausmeister sei in Personalunion „Ein-Mann-Hausbürgerwehr, Türzurammeldienst und Sittenwärter“ (S. 31) und abends zusätzlich „Dämmerungswächter“ (S. 31). Die kreativen Wortschöpfungen und Neologismen bilden das sprachliche Korrelat zur zentralen Stellung von Kommunikation im Roman. In Krankenhäusern fühlt sich Schornstein „immer unglaublich wohl, manchmal sogar überschwänglich glücklich“ (S. 165). Dies hat den einfachen Grund, dass er dort immer „lieb umsorgt wird“ und „frei von Pflichten“ sei, zudem würden ihm die „regelmäßigen Abläufe sehr gut“ (alles S. 165) tun. Auch habe ihm der Herzinfarkt Glück gebracht, was wiederum folgerichtig ist, weil dadurch seine seltene Krankheit erst entdeckt wurde.

Schornstein ist als Ich-Erzähler (logischerweise) der einzige Wahrnehmungsträger, allerdings nimmt er intensiv, genau und mit vielen Sinnen wahr. Es dominieren seine visuellen Wahrnehmungen. Das ,gezielte Beobachten“ von Räumen und Figuren spielt eine zentrale Rolle und hat einen kontrollierenden und überwachenden Charakter. So wird Schornstein zum „Beobachter des Treppenhausgeschehens“ (S. 76), er beobachtet die Trinker-Cliquen und Frau Schwan genau, „alles, was mit ihr und ihren Leuten zusammenhing" (S. 19) und er passiert deshalb auch immer wieder das Rondell. Besonders drastisch ist die mehrfache Beschreibung seiner olfaktorischen Eindrücke in Frau Schwans Wohnung (vgl. S. 74, 127f).

Im Laufe der Zeit ändert sich die Wahrnehmung und Empfindung des Protagonisten fundamental. Dies wird besonders deutlich als einer aus Schornsteins ,Patientengruppe stirbt (vgl. S. 259). Schornstein sieht Menschen, die er früher verachtete - depressive und verunsicherte Menschen - nun als seine „Kumpel und Verbündeten“ (S. 261) an. Seine Wahrnehmung wird sensibilisiert und er nimmt „verstärkt auch fröhlichere Dinge und Menschen wahr“ (ebd.). Wenn er „kurzzeitig eine kleine Euphorie“ (ebd.) in sich spürt, ist er „sofort voll diffuser Zuneigung zu allen“ (ebd.). Seine veränderte Wahrnehmung wirkt sich auf seine Emotionen und schließlich auch auf die Räume aus. Beim Spaziergang „wölbte sich [der Parkplatz] in der Mitte“ und er merkt, „dass die Tiefenverhältnisse in [s]einem Blickfeld nicht stimmten“ (alles S. 263). Sein gesamter Vorstellungs-, Wahrnehmungs- und Empfindungsraum hat sich verändert. Welche Ursachen das hat, bleibt genauso offen wie die Ursache seiner tatsächlichen ,Heilung‘. Schornsteins dynamische Wahrnehmung der Figuren korreliert mit einer veränderten Wahrnehmung des Raumes.

16 Ironisch gemeinte Anspielungen von Schornstein auf den Holocaust mittels imitiertem Nazijargon sind im Roman mehrfach zu entdecken. Auch dadurch zieht Schornstein immer wieder eine Grenze zu den anderen Figuren, insbesondere gegenüber seiner Frau Anne. 


\section{Semantiken des erzählten Raumes}

Eine sowohl konkret als auch metaphorisch betrachtet ,zentrale Stellung“ im Leben des Protagonisten nimmt sein Körper ein. Er ist dem Protagonisten besonders wichtig, auch und gerade, weil er nahezu ständig gesundheitliche Probleme hat. Der ,Körper-Raum ist für Schornstein auch bei anderen Figuren von großer Bedeutung. Deshalb wird auf diesen Aspekt hier noch einmal dezidiert eingegangen. ${ }^{17}$

Die von Schornstein erzählte Geschichte beginnt bereits mit einem ,körperlichem“ Schicksalsschlag, seinem Herzinfarkt. ${ }^{18}$ Seitdem leidet er schier ununterbrochen und mit wechselnder Intensität an physischen und psychischen Problemen. Sein Körper steht deshalb für ihn immer wieder im Fokus seiner Beobachtungen. Ausführlich schildert er, was er im Laufe der letzten zehn Jahre durchlitten hat. Er beschreibt auch intime körperliche Details und Verhaltensweisen bei anderen Figuren: Die strengen, privaten körperlichen ,Reinigungsrituale‘ seiner Mutter kontrastiert er mit dem ungezwungenen, öffentlichen Küssen und dem dabei erfolgten, heimlichen Austausch von Kirschkernen zwischen ihm und seiner Frau (vgl. S. 62). Dadurch versucht er auch, die von seinem Psychiater bei ihm diagnostizierte ,transgenerationelle Traumatisierung، (S. 190) zu entkräften bzw. zu widerlegen.

Der konkrete körperliche Raum der Mundhöhle - metaphorisch gesehen der ,Raum der Sprache - fungierte bei seiner Mutter als besonders sensibler und schützenswerter Raum. Er bildete „eine heilige Sonderputzzone“ (S. 62) und sollte vor ,Verunreinigungen“ - man kann ergänzen ,sowohl konkret-physischer als auch metaphorisch-sprachlicher Art' - freigehalten werden. Umso schockierender wäre für seine Mutter gewesen, Schornstein und seine Frau beim intimen, aber in der Öffentlichkeit durchgeführten „Gut-Kirschen-Essen“ (S. 62) zu beobachten.

Der Protagonist sieht seinen Körper als verletzbaren Raum an, der immer wieder Angriffen von anderen ausgesetzt ist. Mehrfach und massiv wird sein Körper von unfähigem Krankenhauspersonal (vgl. S. 151ff) oder aufgrund von Unachtsamkeit von ihm selbst (vgl. S. 19, 157ff) verletzt. Seine Haut - als ,Grenze‘ zwischen Körper-Innerem und Äußerem - ist ihm „als Schutzschicht viel zu dünn“ (S. 162). Deshalb blute er nach den Behandlungen wie ein „sich selbst schlachtendes Schwein“ (S. 161). Schornstein wehrt sich auch gegen die von Dr. Brakwahrt vorgeschlagene „Körpertherapie“ (S. 186), denn er weiß, dass sein „Körper auf keinen Fall angefasst werden wollte“ (ebd.). Den Kulminationspunkt in der Beschreibung dieses sensiblen und dynamischen Körperraumes bildet die Szene, in der Schornstein unter der Dusche „der Zersetzung [s]eines Körpers beiwohnen“ (S. 266) und seinem „materiellen Ende sozusagen ins Auge sehen konnte“

17 Hier wird deutlich, was es heißt, ,räumlich zu denken‘ (vgl. Bachmann Medick 2009: 292). Es geht nicht nur darum, konkrete Räume zu erfassen, sondern möglichst viele Aspekte des Werkes aus raumnarratologischer Sicht zu analysieren, um eventuell zu neuen Erkenntnissen und Interpretationsansätzen zu gelangen.

18 Auch am Ende des Romans wird der körperliche Aspekt in der Beziehung zwischen Schornstein und Anne noch einmal betont. Schornstein bespricht zusammen mit seiner Frau seine persönliche und berufliche Zukunft und will bzw. soll mit körperlicher Betätigung - Joggen - beginnen. Zudem habe er „auf dem erotischen Sektor [mit seiner Frau Anne] noch viel vor“ (S. 283). 
(S. 267). Dass es sich dabei doch nicht um sein (körperliches) Ende handelt, wird klar, als er anschließend „als rituell erneuerter Mensch“ (ebd.) die Badewanne verlässt. Auch nach weitgehend überstandener Krankheit bleibt der Körper ein zentrales Thema im Roman. Anne beschreibt in ihrer finalen „Ansprache an die Engel“ (S. 280) Schornsteins Körper gleichsam wie in einer Zusammenfassung noch einmal detailliert von oben bis unten (vgl. S. 280ff). Und auch bei ihm selbst bleibt im Rückblick das vertraute körperliche Zusammensein mit seiner Frau - „unsere langwierigen Schmusereien“ (S. 282) als „die am sinnvollsten verbrachte Zeit unseres Lebens“ (S. 282) in Erinnerung. All dies unterstreicht die eminente Bedeutung des Körper-Raumes im Roman.

\section{Fazit}

Zusammenfassend lässt sich feststellen, dass konkrete und metaphorische Räume im Roman eine wichtige, wenn nicht sogar die dominierende Rolle spielen. Die konkreten Makroräume der erzählten Welt mit eindeutig realweltlichem Bezug verleihen den geschilderten Geschehnissen authentischen Charakter, was durch zahlreiche autobiografische Bezüge noch verstärkt wird. Die geschilderten Mikroräume haben eher imaginären Charakter, was dem authentischen Charakter des Romans jedoch keinen Abbruch tut. Bei den Mikroräumen der erzählten Welt handelt es sich in erster Linie um Innen-Räume. Diese Räume bilden gleichzeitig metaphorische Räume ab, sie haben zum Großteil marginalen, transitären, hybriden und teilweise auch heterotopen Charakter. Zuweilen sind sie auch eine Kombination aus obigen Raumcharakteren und multifunktional: So fungieren Räume gleichzeitig als Arbeits- und Wohnraum, Wohn- und Asylraum oder im extremsten Beispiel gleichzeitig als Arbeitsraum, Wartezimmer, Bühne und Zuschauerraum.

Es werden im Roman gleichzeitig zwei Arten von ,Gegen-Räumen' konstruiert: so kann man zwischen Innen- und Außenräumen unterscheiden, was nahezu deckungsgleich ist zur Unterscheidung zwischen privatem und öffentlichem Raum. Diese Unterteilung wird evoziert durch Schilderungen von Handlungen sowohl in diesen Räumen als auch an deren Grenzen, zum Beispiel an Türschwellen von Wohnungen.

Grenzen sind sowohl auf der konkreten als auch der metaphorischen Ebene mehr oder weniger deutlich sichtbar: nicht nur als Grenze zwischen innen und außen oder zwischen öffentlich und privat, sondern auch als sozialgesellschaftliche, sprachliche oder körperliche Grenzen. Alle diese Grenzen werden im Laufe des Romans jedoch überschritten. Einige werden durch die Grenzüberschreitung erst sichtbar, so zum Beispiel die Schamgrenze oder die Grenze des literarischen Geschmacks. Größtenteils ,unerwartete' Grenzüberschreitungen seitens des Protagonisten oder anderer Figuren verwischen damit ihre Trennschärfe und bewirken eine Hybridisierung der Räume. Es kann schließlich nicht mehr genau zwischen öffentlich und privat, innen oder außen unterschieden werden. Räume können auch nicht mehr eindeutig bestimmten Figuren zugeordnet oder klassifiziert werden. Räume werden auf diese Weise dekonstruiert und neu semantisiert. Das Ergebnis sind dynamische, multifunktionale und hybride Räume. 
Zwei konkrete Räume der erzählten Welt sind im Roman dominant und haben gleichzeitig metaphorische Bedeutung. Der Körperraum bzw. der Körper des Protagonisten fungiert als Spiegel der physischen und psychischen Verfasstheit des Protagonisten. Er ist Schwachpunkt und Angriffsziel einerseits, dient aber auch als Mittel, diese Schwäche und Verletzlichkeit zu überwinden. Dabei spielt der Mund(-raum) bzw. die Sprache das wichtigste ,Körper-Instrument‘ für den Protagonisten. Der Raum der Sprache, die schriftliche und mündliche Kommunikation, stellt für den Protagonisten die wichtigste Waffe in seinem Kampf gegen das schier übermächtige Gesundheitssystem dar. Letzteres steht stellvertretend für einen, alle anderen Räume durchdringenden, Machtraum, in dem die Figuren und Handlungen eingeflochten sind. Dieser Machtraum wird vom kranken Protagonisten als willkürlich und undurchschaubar empfunden, aber es ist für ihn (über-) lebenswichtig, sich darin zu behaupten.

Neben dem Körperraum ist der Wohnraum der wichtigste konkrete Raum der erzählten Welt. Die Wohnung dient den Figuren als Rückzugsort, Ort der Sicherheit, der Erholung und des sozialen Austausches. Für den Protagonisten ist die Wohnung ein sehr dynamischer Raum: anfangs Wohn- und Arbeitsraum dient sie später auch als ,Organisations-Zentrum‘, von dem aus er seinen Kampf gegen das Gesundheitssystem führt. Die Wohnung dient dem Protagonisten gewissermaßen als ,Zentrum‘, von dem aus er „ins Leben springen“ (Flusser 1999) kann.

Der Roman wird rückblickend und durchgehend aus der Ich-Perspektive des Protagonisten erzählt. Der Erzähler verweist durch wiederholte metasprachliche Einschübe auf die Subjektivität, Emotionalität und damit Fiktionalität seiner (räumlichen) Beobachtungen. Er nimmt drastisch und mit allen Sinnen wahr, besonders jedoch visuell. Auffallend ist die dynamische Wahrnehmung des Protagonisten, in Folge derer sich die Wertigkeit seiner Wahrnehmungen nahezu umkehrt. Dies bestätigt noch einmal die Subjektivität, Dynamik und Konstruiertheit der erzählten Räume. Insgesamt sind die konkreten Räume der erzählten Welt durch eine Individualität und Originalität gekennzeichnet, die an Skurrilität grenzt, welche mit den Charakteren der darin agierenden Figuren korreliert.

\section{Quellen- und Literaturverzeichnis}

Assmann, Aleida (2006): Erinnerungsräume. München.

Bachmann-Medick, Doris (2009): Cultural turns. Neuorientierungen in den Kulturwissenschaften. Reinbek.

Böhme, Hartmut (2005): Topographien der Literatur. Deutsche Literatur im transnationalen Kontext. Stuttgart.

Bourdieu, Pierre (1991): Physischer, sozialer und angeeigneter physischer Raum. In: Wentz, Martin (Hg.): Stadt-Räume. Die Zukunft des Städtischen. Frankfurt a. Main. S. 25-34.

Bourdieu, Pierre (1999): Die Regeln der Kunst. Genese und Struktur des literarischen Feldes. Frankfurt am Main.

Certeau, Michel de (1988): Bericht von Räumen. In: Die Kunst des Handelns. Berlin. S. $215 f$.

Cornejo, Renata (2016): Heimatbilder und -konstruktionen in Werken der deutschsprachigen 
Autorinnen und Autoren tschechischer Herkunft (Moníkova, Faktor, Fusek). In: Aussiger Beiträge 10, S. 117-134.

Cornejo, Renata (2010): Heimat im Wort. Zum Sprachwechsel der deutsch schreibenden tschechischen Autorinnen und Autoren nach 1968. Eine Bestandsaufnahme. Wien.

Cornejo, Renata (2010): Der zähe Landsmann Franz Kafka im Werk von Libuše Moníková und Jan Faktor. In: Kondrič, Horvat: Franz Kafka und Robert Walser im Dialog. Berlin. S. 381-405.

Dennerlein, Karin (2009): Narratologie des Raumes. Berlin.

Döring, Jörg (2008): Spatial turn. Das Raumparadigma in den Kultur- und Sozialwissenschaften. Bielefeld.

Dünne, Jörg (2015): Handbuch Literatur \& Raum. Berlin; Boston.

Faktor, Jan (2006): Schornstein. Köln.

Foucault, Michel (2005): Analytik der Macht. Frankfurt am Main.

Foucault, Michel (2006): Von anderen Räumen. In: Dünne Jörg et al (Hrsg.): Raumtheorie: Grundlagentexte aus Philosophie und Kulturwissenschaft. Frankfurt am Main. S. 317-329.

Flusser, Vilem (1994): Von der Freiheit des Migranten. Einsprüche gegen den Nationalismus. Köln. (CD-Aufnahme)

Frank, Caroline (2017): Raum und Erzählen. Würzburg.

Günzel, Stephan (2010): Raum. Ein interdisziplinäres Handbuch. Stuttgart.

Hallet, Wolfgang [Hrsg.] (2009): Raum und Bewegung in der Literatur. Bielefeld.

Hausbacher, Eva (2008): Poetik der Migration. Transnationale Literatur zeitgenössischer russischer und kroatischer Autoren. In: WIENER SLAVISTISCHES JAHRBUCH, Band 54, S. 47-62. Wien.

Lefebrve, Henri (1991): The production of space. Oxford, Cambridge.

Löw, Martina (2001): Raumsoziologie. Frankfurt am Main.

Lüdke, Martin (2006): Nach Klassenkampf kommt Kassenkampf! Rezension in „Die Zeit“ vom 18. 6. 2006.

Magenau, Jörg (2006): In überlebensnotwendiger Distanz. In: Wespennest Heft 142, S. 74-81.

Schneider, Wolfgang (2006): Kassenkampf: Jan Faktor nimmt es fröhlich mit den Ärzten auf. Rezension in „Frankfurter Allgemeine Zeitung“ vom 18. 12. 2006.

Warning, Rainer (2015): Utopie und Heterotopie. In: Dünne, Jörg (2015): Handbuch Literatur \& Raum. Berlin; Boston. S. 178-187.

Wichard, Norbert (2012): Mitteleuropäische Blickrichtungen. Geschichtsdarstellung bei Saša Stanišic und Jan Faktor. In: Aussiger Beiträge 6, S. 159-175.

Wiest, Karin (2006). Soziale Grenzen in Städten: Repräsentationen von Wohngebieten in den neuen Ländern. Europa Regional, 14.2006 (1), S. 33-40.

Wilhelmer, Lars (2015): Transit-Orte in der Literatur. Bielefeld.

Mag. Karl-Heinz Gmehling. M. A. / kgmehling@yahoo.de

Univerzita J. E. Purkyně, Filozofická fakulta, Katedra germanistiky

Pasteurova 3571/13, 40096 Ústí nad Labem, Česká republika

This work can be used in accordance with the Creative Commons BY-SA 4.0 International license terms and conditions (https://creativecommons.org/licenses/by-sa/4.0/legalcode). This does not apply to works or elements (such as image or photographs) that are used in the work under a contractual license or exception or limitation to relevant rights 